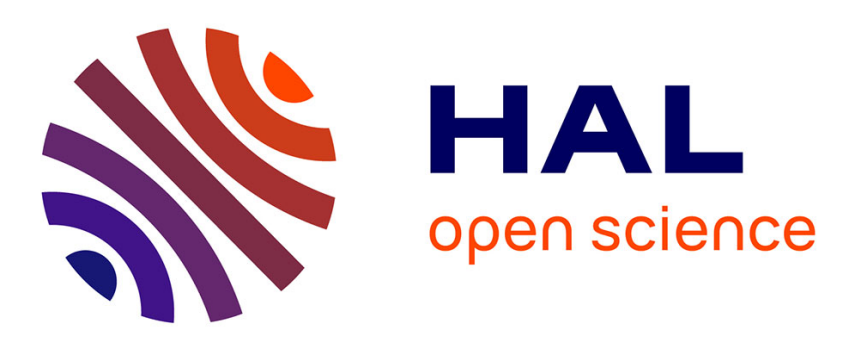

\title{
Détermination de longueurs d'onde spécifiques pour la mesure des glucosinolates du colza par spectrophotométrie de réflexion dans le proche infrarouge
}

\author{
Marc Lila, Vincent Furstoss
}

\section{To cite this version:}

Marc Lila, Vincent Furstoss. Détermination de longueurs d'onde spécifiques pour la mesure des glucosinolates du colza par spectrophotométrie de réflexion dans le proche infrarouge. Agronomie, 1986, 6 (8), pp.703-707. hal-00884927

\section{HAL Id: hal-00884927 \\ https://hal.science/hal-00884927}

Submitted on 1 Jan 1986

HAL is a multi-disciplinary open access archive for the deposit and dissemination of scientific research documents, whether they are published or not. The documents may come from teaching and research institutions in France or abroad, or from public or private research centers.
L'archive ouverte pluridisciplinaire HAL, est destinée au dépôt et à la diffusion de documents scientifiques de niveau recherche, publiés ou non, émanant des établissements d'enseignement et de recherche français ou étrangers, des laboratoires publics ou privés. 


\section{Détermination de longueurs d'onde spécifiques pour la mesure des glucosinolates du colza par spectrophotométrie de réflexion dans le proche infrarouge}

Marc LILA \& Vincent FURSTOSS

I.N.R.A., Station d'Amélioration des Plantes fourragères, F 86600 Lusignan

\begin{abstract}
La spectrophotométrie de réflexion dans le proche infrarouge présente, grâce à sa rapidité, un grand intérêt pour la sélection qualitative de diverses espèces. Une étude a été réalisée pour le dosage des glucosinolates totaux du colza graines entières (Brassica napus L.) à partir de 5 populations différentes. Les équations d'étalonnage établies pour chaque population montrent qu'il existe 5 longueurs d'onde spécifiques pour ce constituant : 1674 $1660-1618$ - 1650 et $1632 \mathrm{~nm}$ par ordre croissant d'importance. Les erreurs de prédiction se situent entre 7,8 et $15,6 \mu \mathrm{mole} / \mathrm{g}$ selon les populations. Un test de vérification a permis de montrer que cette erreur se maintient au même niveau en faisant varier les longueurs d'onde de $\pm 4 \mathrm{~nm}$. Après fabrication des filtres spécifiques, il sera possible d'utiliser un appareil de routine (roue de filtres) pour le dosage rapide et simultané des protéines, de l'huile et des glucosinolates des graines entières de colza.
\end{abstract}

Mots clés additionnels : Brassica napus L., graines entières.
Choice of specific wavelengths for glucosinolate analysis in whole rapeseed by near infrared reflectance spectroscopy.

Near infrared reflectance spectroscopy is a really attractive rapid method for quality breeding in many species. After successful calibrations for oil and protein in whole rapeseed the method was also tried for glucosinolate content. This was carried out on five different populations. The optimal wavelengths selected (1 $674-1660$ $1618-1650-1632 \mathrm{~nm}$ ) for the most representative population gave adequate predictions for the other four but it was impossible to use the same equation for glucosinolate analysis on samples harvested in different years. For three populations we had to do a bias and slope adjustment, and for the last we had to adjust each coefficient of the equation. The standard error of the prediction varied from 7.8 to $15.6 \mu$ mole/g while the reference error of analysis was about $8 \mu \mathrm{mole} / \mathrm{g}$. To avoid large increases in error due to wavelength variations each population was tested separately. Each wavelength was varied by $\pm 4 \mathrm{~nm}$, without affecting the standard error. Specific filters must be now manufactured so as to use routine apparatus (with a Technicon 400 filter wheel) to analyse proteins, oils and glucosinolates simultaneously on whole rapeseed.

Additional key words : Brassica napus $L$, whole rapeseed.

\section{INTRODUCTION}

L'intérêt de la spectrophotométrie de réflexion dans le proche infrarouge en sélection pour la qualité des céréales et des oléagineux a déjà été démontré par de nombreux auteurs : NORRIS \& HART (1965), puis HYMOWITZ et al. (1974), NORRIS et al. (1976), ALLISON et al. (1978), RUBENTHALER \& BRUINSMA (1978), WILliAMS et al. (1978), GILL et al. (1979) et TKACHUK (1981) entre autres. Après étalonnage, l'analyse est rapide, multiparamètre et non destructive si l'on peut travailler sur graines entières. Le travail présenté ici a consisté à rechercher s'il était possible de prédire la teneur en glucosinolates totaux du colza graines entières malgré l'échec relatif constaté par TKACHUK (1981). Ce dernier a pu réaliser des étalonnages pour la détermination sur graines entières de l'huile et des protéines, nous avons nous-mêmes constaté que ce travail ne présente pas de difficultés particulières et que les résultats sont fiables. La sélection de variété à 
faible teneur en glucosinolates dans le tourteau reste un objectif important pour le sélectionneur qui ne dispose que de tests peu précis (test glucose, test palladium) ou de l'analyse par chromatographie en phase gazeuse THIES (1976), précise, mais longue et coûteuse. On comprend donc les avantages que pourrait apporter la réalisation d'un étalonnage fiable sur un appareil infrarouge de routine (appareil à filtres).

\section{MATÉRIEL ET MÉTHODES}

\section{A. Matériel de mesure}

Le matériel infrarouge est constitué d'un spectrophotomètre "Infra-alyser Technicon 500", piloté par un calculateur HP 1000 A 600 qui, grâce à son réseau holographique peut balayer le spectre de 550 à $2650 \mathrm{~nm}$, et d'un spectrophotomètre "Infra-alyser Technicon 400" piloté par un calculateur HP 85. Cet appareil ne possède qu'une roue de 19 filtres et sert à la réalisation des analyses de routine. Le constructeur propose un choix parmi une trentaine de filtres pour équiper ce spectrophotomètre. Le logiciel implanté sur le calculateur HP 1000 offre 2 algorithmes de recherche des longueurs d'onde. L'un effectue ce que l'on appelle une régression montante (nommée "STEP UP'), l'autre (appelé “COMBO") permet de tester toutes les combinaisons de $\mathrm{n}$ longueurs d'onde parmi 100 maximum, n étant inférieur à 6 essentiellement pour limiter les temps de calcul.

\section{B. Matériel végétal}

Nous avons disposé pour notre étude de 5 populations de colza (Brassica napus L.) issues de lieux et d'années différentes selon la description ci-dessous :

\section{- population 1 :}

54 échantillons récoltés en France par le Centre Technique Interprofessionnel des Oléagineux Métropolitains (CETIOM) en 1984, comprenant des origines génétiques différentes. On a collecté 2 spectres par échantillon. La teneur en glucosinolates totaux variait de 6,80 à $220,50 \mu$ mole $/ g$; la moyenne était de 99,22 et la déviation standard de 61,39 . Les échantillons de cette population ont été soigneusement choisis pour obtenir une variabilité maximum représentant au mieux ce qui peut être rencontré actuellement dans une pépinière de sélection en ce qui concerne la teneur en glucosinolates ;

\section{- population 2 :}

33 échantillons récoltés à Rennes en 1983, d'origines génétiques différentes ; 2 spectres par échantillon ; teneur en glucosinolates totaux variant de 148,20 à $255,20 \mu \mathrm{mole} / \mathrm{g} ;$ moyenne $=183,83$; déviation standard $=24,82$;

\section{- population 3 :}

30 échantillons récoltés en Suède, aimablement fournis par la Société Technicon ; 2 spectres par échantillon; teneur en glucosinolates totaux variant de 18,10 à $122,30 \mu$ mole/g ; moyenne $=57,12$; déviation standard $=33,46$;

\section{- population 4 .}

30 échantillons récoltés à Lusignan en 1983 et représentant une seule variété ayant subi des niveaux différents d'infestation de charançon des siliques ( $\mathrm{Ceu}$ thorrhynchus assimilis) ; 2 spectres par échantillon ; teneur en glucosinolates totaux variant de 150,18 à $222,00 \mu \mathrm{mole} / \mathrm{g} ;$ moyenne $=190,56$; déviation standard $=17,92$;

- population 5 :

20 échantillons récoltés en 1982, d'origines génétiques et géographiques différentes; 3 spectres par échantillon ; teneur en glucosinolates totaux variant de 31,60 à $175,60 \mu \mathrm{mole} / \mathrm{g} ;$ moyenne $=96,66$; déviation standard $=41,70$.

Les analyses de référence réalisées en différents laboratoires selon l'origine des populations (Station d'Amélioration des Plantes de Rennes, CETIOM, Suède) par chromatographie en phase gazeuse (THIES, 1976) ont une précision de l'ordre de $8 \mu \mathrm{mole} / \mathrm{g}$.

Les valeurs spectrales des graines entières ont été collectées après passage à l'étuve à $60^{\circ} \mathrm{C}$ pendant $24 \mathrm{~h}$. Le volume de graines mises dans la capsule de mesure était constant et déterminé de façon à maintenir les graines immobiles.

\section{Méthodes}

Les valeurs spectrales des populations 2 et 3 ont été collectées simultanément sur l'IA 400 et l'IA 500. Celles-ci ont permis de tester si les équations calculées à partir des valeurs spectrales de l'IA 500 sont transférables sur l'IA 400. Pour les populations 1, 4 et 5, la collecte n'a été effectuée que sur l'IA 500.

La recherche d'une équation utilisant les longueurs d'onde des 30 filtres disponibles sur IA 400 a été réalisée en réunissant les valeurs spectrales des populations 5 et 2. L'algorithme de sélection des longueurs d'onde nous a permis de tester toutes les combinaisons possibles de $n$ longueurs d'onde parmi $30, n$ variant de 3 à 7 .

Aucune équation appliquée sur des échantillons inconnus (n'ayant pas servi au calcul de régression) n'a donné de prédiction satisfaisante. Ainsi une équation calculée à partir des longueurs d'onde fournies couramment par le constructeur n'a pu être transférée sur l'IA 400. Les meilleurs résultats ont été obtenus en utilisant la population 1, préparée avec soin, comme lot de calibration. Les 2 algorithmes de sélection des longueurs d'onde ont également été testés. La technique "Combo" s'est révélée supérieure (meilleur coefficient de corrélation multiple).

Pour appliquer celle-ci, le nombre de valeurs spec trales par échantillon de la population 1 a été réduit de 350 à 100 , en augmentant l'incrément de 4 à $14 \mathrm{~nm}$. Pour réduire les temps de recherche, nous avons également supprimé le calcul de certaines combinaisons redondantes. Grâce à cela, le calculateur a pu déterminer en $24 \mathrm{~h}$ la meilleure combinaison de 5 longueurs d'onde parmi 100. Ces 5 longueurs d'onde sont toutes situées dans la zone 1600 à $1680 \mathrm{~nm}$. Le choix a été affiné en restant dans cette zone mais en diminuant le pas de 14 à $4 \mathrm{~nm}$. L'équation ainsi obtenue a été appliquée sur les populations $2,3,4$ et 5 afin d'obtenir des valeurs de prédictions (fig. 1). 

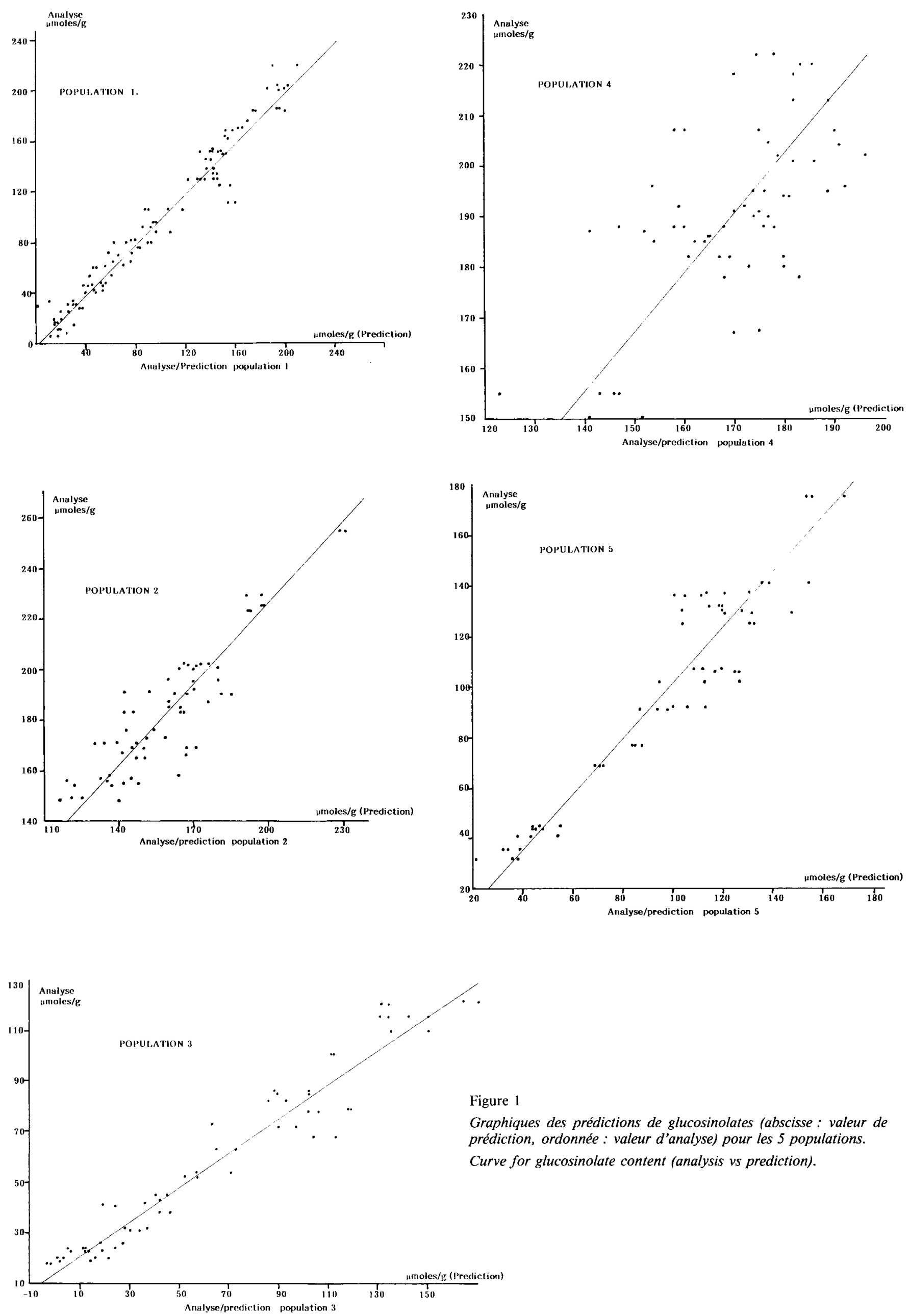

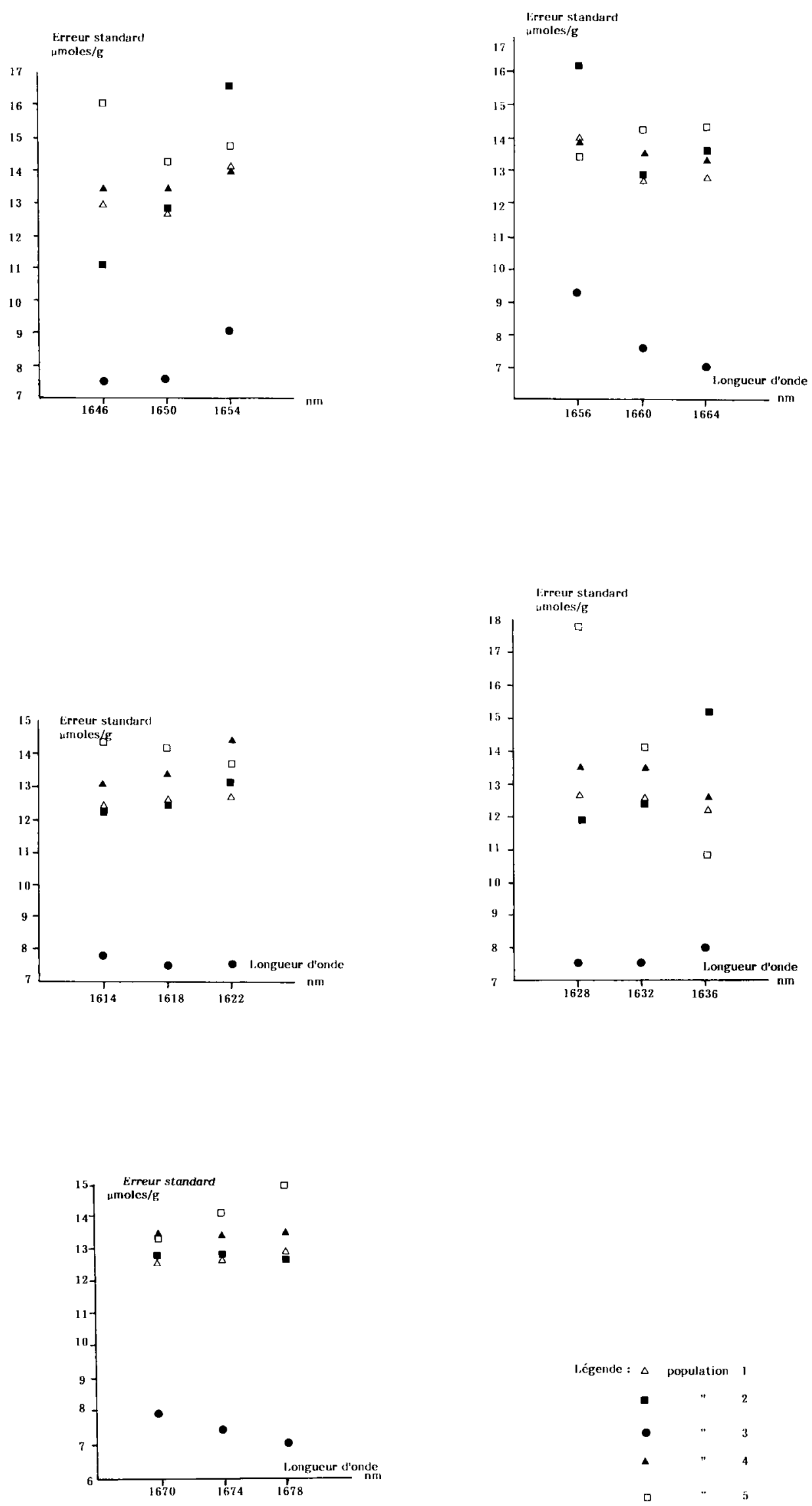

Figure 2

Evolution de l'erreur standard estimée autour des 5 longueurs d'onde de calibration (variation $\pm 4 \mathrm{~nm}$ ).

Standard error of estimate in relation to calibration wavelength (variation $\pm 4 \mathrm{~nm}$ ). 


\section{RÉSULTATS ET DISCUSSION}

On note, à la lecture du tableau 1 , que les erreurs standards de prédiction ainsi que les coefficients de corrélation sont d'un niveau très acceptable pour toutes les populations sauf pour la population 4 , mais celle-ci présente 2 défauts : une faible variabilité des teneurs et une culture en conditions particulières (sous cages).

\section{TABLEAU 1}

Valeurs des paramètres d'étalonnage des glucosinolates totaux, obtenues pour les 5 populations de colza (déviation et erreur exprimées en umole/g).

Parameters for glucosinolate calibration, obtained on five populations of whole rapeseed (standard deviation and standard error in umole/g).

\begin{tabular}{lccccc}
\hline \hline \multicolumn{1}{c}{ Numéro population } & 1 & 2 & 3 & 4 & 5 \\
\hline Déviation standard population & 61,4 & 24,8 & 33,5 & 17,9 & 41,7 \\
\hline Erreur standard prédiction & 12,4 & 11,4 & 7,8 & 15,6 & 14,1 \\
\hline Coefficient corrélation & 0,98 & 0,90 & 0,97 & 0,63 & 0,94 \\
\hline Nombre de spectres & $2 \times 54$ & $2 \times 33$ & $2 \times 30$ & $2 \times 30$ & $3 \times 20$ \\
\hline \hline
\end{tabular}

L'objectif étant d'obtenir une erreur de prédiction acceptable pour le sélectionneur soit entre 12 et $14 \mu \mathrm{mole} / \mathrm{g}$, nous avons considéré que ce dernier choix de longueurs d'onde était satisfaisant et nous avons donc retenu par ordre croissant d'importance pour la signification des coefficients de régression les valeurs suivantes : $1674-1660-1618-1650$ et $1632 \mathrm{~nm}$; à noter que l'on se situe dans la même zone du spectre que TKACHUK (1981). Certains de ces filtres $(1618,1632)$ ne sont pas disponibles, il faut donc en demander la fabrication. Sachant qu'il existe 2 qualités de filtres interférentiels, l'une ayant une précision de centrage de la longueur d'onde à $\pm 2 \mathrm{~nm}$, l'autre à $\pm 5 \mathrm{~nm}$, il faut lever l'incertitude quant à la précision à demander. La conséquence d'un centrage peu précis peut conduire à une augmentation importante de l'erreur de prédiction par suite d'une variation notable du spectre de corrélation autour de la longueur d'onde déterminée. Nous avons procédé à un test de variation de longueur d'onde autour de la valeur centrale, c'est-à-dire que, pour chaque popula- tion de colza, nous avons calculé une équation en faisant varier à tour de rôle chacune des 5 longueurs d'onde de plus ou moins $4 \mathrm{~nm}$. Nous avons ainsi obtenu 11 équations ainsi que la valeur de l'erreur estimée. Les résultats présentés dans les 5 graphiques de la figure 2 montrent en général une bonne stabilité de la valeur moyenne pour la longueur d'onde centrée. Il semble donc qu'en respectant une relative rigueur quant à la fabrication des filtres interférentiels ( $\pm 2 \mathrm{~nm}$ pour les longueurs d'onde 1632 et 1650 ), on puisse espérer maintenir un niveau d'erreur acceptable et obtenir ainsi un transfert d'étalonnage sur IA 400 permettant en routine la prédiction des glucosinolates du colza.

\section{CONCLUSION}

Le travail réalisé permet de penser que le choix des longueurs d'onde est bon, car l'étalonnage a été confirmé sur d'autres populations issues de lieux et d'années divers. Toute nouvelle population d'origine différente (lieu, année) entraînera au pire une modification des coefficients attribués aux longueurs d'onde sélectionnées. Lorsque les contraintes matérielles concernant la fabrication de filtres spécifiques seront levées, il sera possible de prédire avec une précision suffisante la teneur en glucosinolates totaux du colza, simultanément avec les teneurs en protéines et huiles à un rythme d'analyse très élevé.

Ces résultats encourageants laissent à penser qu'il ne serait pas vain de tenter de réaliser des étalonnages plus spécifiques : glucosinolates indoliques par exemple, à la condition que ces composés ne soient pas fortement corrélés avec les glucosinolates totaux (cas de la progoitrine $: r=0,98$ ). On peut également envisager de développer un étalonnage pour des populations à faible teneur en glucosinolates totaux.

Reçu le 8 janvier 1985. Accepté le 11 avril 1986.

\section{REMERCIEMENTS}

Nous remercions M. Renard (I.N.R.A., Rennes), M. Ribailler (CETIOM) et la société Technicon d'avoir fourni certains lots d'échantillons accompagnés des analyses de référence.

\section{RÉFÉRENCES BIBLIOGRAPHIQUES}

Allison M. J., Cowe I. A., McHale R., 1978. The use of infrared reflectance for the rapid estimation of the soluble $\beta$-glucan content of barley. J. Inst. Brewing, 84, 153.

Gill A. A., Starr C., Smith D. B., 1979. Lysine and nitrogen by infrared reflectance analysis as an aid to barley breeding. $J$. Agric. Sci. Camb., 93, 727.

Hymowitz T., Dudley J. W., Collins F. I., Brown C. M., 1974. Estimation of protein and oil concentration in corn, soybean and oatseed by near infrared light reflectance. Crop Sci., 14, 173.

Norris K. H., Hart J. R., 1965. Direct spectrophotometric determination of moisture content of grain and of seeds. Proc. $1963 \mathrm{Int}$. Symp. on humidity and moisture. Principles and methods of measuring moisture in liquids and solids., 4, 19-25.
Norris K. H., Barnes R. F., Moore J. E., Shenk J. S., 1976. Predic ting forage quality by infrared reflectance spectroscopy. J. Anim. Sci., 43, 889 .

Rubenthaler G. L., Bruinsma B. L., 1978. Lysine estimation in cereals by near infrared reflectance. Crop Sci., 18, 1039.

Thies W., 1976. Quantitative gas liquid chromatography of glucosinolates on a microliter scale. Fette, Seifen, Austrichm, 78, 231-234.

Tkachuk R., 1981. Oil and protein analysis of whole rapeseed kernels by near infrared reflectance spectroscopy. J.A.O.C.S., August 1981,819 .

Williams P. C., Stevenson S. G., Starkey P. M., Hawtin G. C., 1978. The application of near infrared spectroscopy to protein testing on pulse breeding program. J. Sci. Food Agric., 29, 285. 